\title{
A Case of Abnormal Lymphatic-Like Differentiation and Endothelial Progenitor Cell Activation in Neovascularization Associated with Hemi-Retinal Vein Occlusion
}

\author{
Sirpa Loukovaara ${ }^{a}$ Erika Gucciardoc ${ }^{c}$ Pauliina Repo ${ }^{c}$ Jouko Lohi ${ }^{\text {b }}$ \\ Petri Salven $^{\mathrm{b}}$ Kaisa Lehti ${ }^{\mathrm{c}}$ \\ ${ }^{a}$ Unit of Vitreoretinal Surgery, Department of Ophthalmology, and ${ }^{b}$ Department of \\ Pathology, Haartman Institute, Helsinki University Hospital, University of Helsinki, and \\ 'Genome-Scale Biology Research Program, Research Programs Unit, Biomedicum Helsinki, \\ University of Helsinki, Helsinki, Finland
}

\section{Key Words}

Hemi-retinal vein occlusion - Endothelial progenitor cell · Platelet endothelial cell adhesion molecule 1 - Podoplanin · Prospero-related homeobox gene 1 - Lymphatic endothelial cell · Lymphatic vessel endothelial hyaluronan receptor $1 \cdot$ Immunohistochemistry · Proliferative diabetic retinopathy . Vascular endothelial stem cell

\begin{abstract}
Purpose: Pathological vascular differentiation in retinal vein occlusion (RVO)-related neovessel formation remains poorly characterized. The role of intraocular lymphatic-like differentiation or endothelial progenitor cell activity has not been studied in this disease. Methods: Vitrectomy was performed in an eye with hemi-RVO; the neovessel membrane located at the optic nerve head was removed and subjected to immunohistochemistry. Characterization of the neovascular tissue was performed using hematoxylin and eosin, $\alpha$-smooth muscle actin, and the pan-endothelial cell (EC) adhesion molecule CD31. The expression of lymphatic EC markers was studied by lymphatic vessel endothelial hyaluronan receptor 1 (LYVE-1), podoplanin (PDPN), and prospero-related homeobox protein 1 (Prox-1). Potential vascular stem/progenitor cells were identified by active cellular proliferation (Ki67) and expression of the stem cell marker CD117. Results: The specimen contained blood vessels lined by ECs and
\end{abstract}


Loukovaara et al.: A Case of Abnormal Lymphatic-Like Differentiation and Endothelial Progenitor Cell Activation in Neovascularization Associated with Hemi-RVO

surrounded by pericytes. Immunoreactivity for LYVE-1 and Prox-1 was detected, with Prox-1 being more widely expressed in the active Ki67-positive lumen-lining cells. PDPN expression was instead found in the cells residing in the extravascular tissue. Expression of the stem cell markers CD117 and Ki67 suggested vascular endothelial progenitor cell activity. Conclusions: Intraocular lymphatic-like differentiation coupled with progenitor cell activation may be involved in the pathology of neovessel formation in ischemia-induced human hemi-RVO.

(C) 2015 S. Karger AG, Basel

\section{Introduction}

Retinal vein occlusion (RVO) is a multifactorial microvascular vitreoretinal eye disease, with complex mechanical, hemodynamic, and rheological shear stress, coagulation, as well as biochemical and immunological factors involved in its pathogenesis.

Regarding the pathogenesis of RVO, various proinflammatory cytokines and angiogenic growth factors induced by hypoxia have been implicated in increased microvascular permeability, inflammation, and fibroproliferation by controlling the inner blood-retinal barrier breakdown, extracellular matrix cytoskeletal remodeling, and endothelial-mesenchymal cell transition. Especially, the presence of ischemia-regulated growth factors, such as vascular endothelial growth factor (VEGF) and angiopoietin 2, as well as of matrix metalloproteinases has been implicated in the pathogenesis of RVO [1].

In adults, pathological angiogenesis and lymphangiogenesis are dynamic hierarchical biological processes related to tissue ischemia/hypoxia in numerous conditions, such as cancer, inflammatory diseases, and various eye disorders [2-8]. In pathological conditions, the secondary ingrowth of lymphatic vessels can also occur in physiologically alymphatic parts of the eye [7]. In lymphatic vasculature, the differentiating lymphatic endothelial cells (LECs) are known to express lymphatic vessel endothelial hyaluronan receptor 1 (LYVE-1), podoplanin (PDPN), and prospero-related homeobox protein 1 (Prox-1) [9-11], showing phenotypic variation according to anatomic location and functional state. Interestingly, solitary adult blood ECs (BECs) have the capability of clonal expansion, and some of the enddifferentiated, mature vascular wall ECs have the potential to acquire stem cell-like characteristics in vivo in angiogenic disorders by turning on the adult stem/progenitor cell CD117 (c-kit) expression [12].

In our study, we performed vitrectomy for the management of end-stage hemi-RVO coupled with vitreous hemorrhage (VH), fulminant intraocular neovascularization, and tractional retinal detachment (TRD). We hypothesized that the natural development of hemiRVO could rely on ischemia-induced lymphatic-like differentiation and adult vascular endothelial stem cell (VESC) proliferation. Therefore, we investigated by immunohistochemistry (IHC) the pathogenesis of RVO-related neovessel formation focusing on multiple known lymphatic markers (LYVE-1, PDPN, and Prox-1), the vascular stem cell marker CD117 (c-kit), and the marker for active cell proliferation Ki67. Our study is considered important since the development of a treatment armamentarium for RVO remains a priority. To develop more effective treatments, it is imperative that we better understand the mechanisms involved in RVO neovessel formation. 
Loukovaara et al.: A Case of Abnormal Lymphatic-Like Differentiation and Endothelial Progenitor Cell Activation in Neovascularization Associated with Hemi-RVO

\section{Patient and Methods}

The study was conducted according to the principles of the Declaration of Helsinki, and it was approved by the Institutional Review Board of Helsinki University Central Hospital and its Ethical Committee. Signed informed consent was obtained from the participant before inclusion into the study.

We present a 58-year-old Caucasian woman who had hypertension, hyperlipidemia, and hypothyreosis. Her medication included metoprolol, simvastatin, acetylsalicylic acid, and thyroxin. The patient was examined in May 2011 in a private eye hospital when her visual acuity (VA) was still full at 1.0 in both eyes. Thereafter, she did not have regular ophthalmic controls until she developed acute painless central VA loss in her left eye in August 2012. In the private eye hospital, biomicroscopic examination revealed inferior hemi-RVO with macular edema (ME) and minor VH. The patient was admitted to the university eye clinic for laser treatment.

On presentation at the Helsinki University Hospital eye clinic in September 2012, her VA in the left eye had decreased to finger counting at $1 \mathrm{~m}$, with tension measured by applanation tonometry being normal at $13 \mathrm{~mm} \mathrm{Hg}$. Her systemic blood pressure was elevated at 189/88 mm Hg. Slit-lamp examination of the anterior segment was normal, and the crystalline lens was clear. On dilated fundus examination, dense $\mathrm{VH}$ prevented an examination of the fundus structural details and also prevented documentation with fluorescence angiography and optical coherence tomography. Ocular ultrasonography revealed some elevation in the inferior part of the retina.

In October 2012, the left eye underwent 23-gauge vitrectomy (Constellation Vision System, Alcon Laboratories, Inc., Fort Worth, Tex., USA). Perioperatively, the patient was found to have hemi-RVO with fulminant floating neovessel formation at the optic disc and ME with epiretinal fibrosis membrane situated in the macular area and along the inferior temporal retinal vascular arcade together with neovascularization as well as local TRD (size of 3 disc diameters). The neovessel formation at the optic disc was excised and removed with microforceps (MAXGrip, Alcon Laboratories) for IHC analysis. Epiretinal and internal limiting membrane peeling was performed, and the ischemic inferior retinal hemisphere was laser photocoagulated at 180 degree with confluent spots. Finally, the eye was filled with C3F8 (octafluoropropane) endotamponade, and the patient was seen 1 week after surgery to attach the detached retina.

Postoperatively, at the final follow-up visit in November 2012, the VA was 0.4 on the Snellen chart, and the intraocular pressure was normal at $14 \mathrm{~mm} \mathrm{Hg}$. Optical coherence tomography revealed slight cystic ME $(270 \mu \mathrm{m})$. The inferior temporal retina was fully attached, and the photocoagulation had been well performed. Her right eye was normal, with full 1.0 VA on the Snellen chart and normal fundus details.

\section{Antibodies and Reagents}

Rabbit polyclonal antibodies against LYVE-1 (Abcam; 1:100) and Ki67 (NCL-Ki67p, Leica Microsystems; 1:3,000), mouse monoclonal antibodies against CD117 (K45, Thermo Scientific; 1:100), $\alpha$-SMA (1A4, Sigma-Aldrich; 1:1,000), PDPN (D2-40, Ventana ready-to-use reagent 760-4395, Roche), and CD31 (JC70A, Dako; 1:50), as well as goat polyclonal antibodies against Prox-1 (R\&D Systems; 1:40) were used.

\section{Immunohistochemistry}

IHC was done essentially as described in our previous report [8]. Briefly, the hemi-RVO neovascular membrane was fixed with $4 \%$ paraformaldehyde for $3 \mathrm{~h}$, immediately after sur- 
Loukovaara et al.: A Case of Abnormal Lymphatic-Like Differentiation and Endothelial Progenitor Cell Activation in Neovascularization Associated with Hemi-RVO

gical excision, washed in PBS, dehydrated, and embedded in paraffin. Serial $5-\mu \mathrm{m}$ sections were cut from each specimen. Standard hematoxylin and eosin (H\&E) staining was employed on each first and last section, before and after a series of sections had been cut for IHC staining. For IHC, sections were deparaffinized in TissueClear (Tissue-Tek) and rehydrated in graded ethanol series. Antigen retrieval was performed by boiling the tissue sections in sodium citrate buffer or by treatment with $0.025 \%$ trypsin for $30 \mathrm{~min}$ at $+37^{\circ} \mathrm{C}$. Sections were subsequently incubated for $10 \mathrm{~min}$ in $0.6 \%(\mathrm{v} / \mathrm{v})$ hydrogen peroxide. Tissue sections were then rinsed and incubated with blocking buffer $(2.5 \%$ normal horse serum; Vector Laboratories) for $30 \mathrm{~min}$. Pretitrated dilutions of primary antibodies were incubated for $2 \mathrm{~h}$ in blocking buffer, followed by incubation with peroxidase-conjugated anti-mouse Ig or anti-rabbit Ig secondary antibodies for $30 \mathrm{~min}(\alpha$-SMA and CD31) or biotinylated anti-mouse Ig or anti-rabbit Ig secondary antibodies (LYVE-1, Prox-1, CD117, and Ki67). For the staining of LYVE-1, Prox-1, CD117, and Ki67 were incubation with peroxidase-labeled streptavidin (Vectastain; Vector Laboratories) for $30 \mathrm{~min}$. Subsequently, the sections were incubated for 10 min with biotinylated tyramine, followed by a 30 -min incubation with peroxidase-labeled streptavidin. Staining was developed by incubation with 3-amino-9-ethyl-carbazole solution (LYVE-1, Prox-1, CD117, and Ki67) or with chromogen substrate diaminobenzidine (Vectastain $\mathrm{ABC}$ kit) according to the manufacturer's instructions. After washes, slides were counterstained with hematoxylin (Sigma), dehydrated, and mounted using Cytoseal (for SMA and CD31; Electron Microscopy Sciences) or directly mounted with Aquamount mounting medium (for LYVE-1, Prox-1, and CD117). Immunofluorescence of paraffin sections was performed using Alexa 488 and Alexa 594 conjugated secondary antibodies, counterstained with DAPI, and mounted with Immumount mounting medium. Light micrographs were taken using a Leica DMLB microscope combined with a computer-controlled Olympus digital camera and Studio Lite software. N Plan 5×, BF, 0.12 NA, N Plan 10×, BF, 0.25 NA, N Plan 20×, $\mathrm{BF}, 0.4 \mathrm{NA}$, and N Plan 40×, BF, $0.65 \mathrm{NA}$ objectives were used. Epifluorescence micrographs of immunofluorescence staining were obtained using Zeiss Axio Imager Z2 upright epifluorescence microscopes with a Plan-Apochromat 40×, 0.75 NA, objective lens. Postacquisition image processing was performed using Corel Draw software X5. Brightness and contrast were linearly adjusted using Corel Photo-Paint X5.

The IHC slides were independently evaluated under a microscope by four investigators (P.R., E.G., K.L., and P.S.) and an experienced pathologist (J.L.). Five non-diabetic epiretinal or subretinal membranes were used as negative controls and melanoma sections as positive controls for CD31 and LYVE-1, essentially as described in our previous study [8]. In addition, CD31, PDPN, and Prox-1 antibody specificities were confirmed on venolymphatic malformation of the human lip, containing dilated capillaries, small venules, and PDPN-positive slitlike lymphatic capillaries (fig. 2c).

\section{Results}

First, we examined cellular components and morphological features of the specimen by IHC and histological analysis in light microscopy. Corresponding histological whole-tissue H\&E staining showed a fibrotic state, with capillary-like vascular structures (fig. 1a). The hemi-RVO specimen displayed neovessels lined by a layer of CD31-positive BECs, and these BECs were covered by $\alpha$-SMA-positive pericytes or smooth muscle cells (fig. 1b). 
Loukovaara et al.: A Case of Abnormal Lymphatic-Like Differentiation and Endothelial Progenitor Cell Activation in Neovascularization Associated with Hemi-RVO

\section{Lymphatic-Like Differentiation in Hemi-RVO Specimen}

Next, we investigated possible lymphatic vessel involvement or potential LEC differentiation by IHC for LEC markers LYVE-1, PDPN, and Prox-1 (fig. 2). Lymphatic-like capillary structures stained positive with LYVE-1 antibody (fig. 2a). Immunoreactivity for PDPN was not found in any vascular structures in our tissue but only outside lumen-like structures, representing potentially inflammatory macrophages or bone marrow-derived cells (fig. $2 \mathrm{a}$, right inset) [13].

Interestingly, nuclear staining of lymphatic-specific transcriptional factor Prox-1 was observed in many CD31-positive lumen-lining ECs and adjacent cells (fig. 2b). Considering the Prox-1 detection also in non-ECs, the specificity of detection was confirmed using venolymphatic malformation of the human lip. As expected, slit-like PDPN-expressing lymphatic capillaries showed positivity for Prox-1 (fig. 2c). Notably, coimmunostaining of Prox-1 and Ki67 showed active proliferation of Prox-1-positive lumen-lining cells (fig. 2d).

\section{Neovascular Hemi-RVO Specimen Displayed Markers of Endothelial Stem/Progenitor}

Cells and Active Cell Proliferation

To estimate the extent of the neoangiogenic process and stem cell-like properties in the hemi-RVO tissue, we analyzed the expression of the proliferation marker Ki67 and the stem cell marker CD117 (c-kit; fig. 3). CD117 positivity was found in CD31-positive capillaries. With larger magnification, CD117-positive cytoplasmic staining was detected in a large proportion of lumen-lining ECs. Additionally, immunoreactivity for the active proliferation marker Ki67 could be detected both in lumen-lining cells and, extravascularly, in our specimen. Thus, the expression of the stem cell marker CD117 and Ki67 is consistent with VESC activity.

\section{Discussion}

This descriptive, morphologic, immunohistochemical study indicates that some intraocular lymphatic-like EC differentiation and VESC activity occur in the end-stage ischemic hemi-RVO neovessel formation. Our unique neovessel specimen was considered to represent the natural outcome of hemi-RVO since no intervening confounding treatment such as laser photocoagulation or intravitreal anti-VEGF agent had been given to the patient before vitreoretinal microsurgery. Our novel findings support the potential involvement of LEC-like endothelial differentiation towards LEC signature together with stem cell activation in RVO pathogenesis, suggesting that the natural antilymphangiogenic mechanisms are overwhelmed in sight-threatening forms of RVO.

By IHC, we could detect both LYVE-1- and Prox-1-positive vessel structures in our hemiRVO neovessel specimen. We focused on these LEC markers since they were known to most plausibly define the lymphatic vessels versus blood vessels $[10,14]$. In our study, the excised hemi-RVO neovessel tissue specimen expressed both the best-characterized LEC marker LYVE-1 as well as the master regulator of lymphatic development, the nuclear transcription factor Prox-1. Thus, the lymphatic-like vessels observed in our hemi-RVO specimen could represent the true development of lymphatic-like vessels, and Prox-1 could serve as the inducer of lymphatic reprogramming of BECs to LECs [6]. Physiologically, lymphatic-like vessels are needed for tissue drainage (homeostasis), metabolism, and immune responses [4]. In pathological fibrovascular RVO eye condition, lymphatic endothelial-like differentiation would occur as a tissue response to growing fluid and metabolic imbalance. 
Loukovaara et al.: A Case of Abnormal Lymphatic-Like Differentiation and Endothelial Progenitor Cell Activation in Neovascularization Associated with Hemi-RVO

Until now, the potential role of PDPN in pathological lymphangiogenesis in adult vascular RVO has not been well explored. Although, according to previous studies, PDPN is known to be one of the most highly expressed genes in lymphatic endothelium [2], it was not expressed in lumen-lining ECs in our hemi-RVO tissue sample, but rather in extravascular cells. While PDPN has been reported to be expressed also by e.g. inflammatory macrophages, the identity of these cells, as well as the reason for the lack of luminal PDPN expression, remain subjects for future studies [13]. Probably, the developmental and/or the functional state of our hemi-RVO neovessels could relate to the lack of luminal PDPN expression, suggesting that the lymphatic vessels remained immature [2]. That could explain why our hemi-CRVO tissue specimen did not display all features of terminally differentiated lymphatic endothelium. Interestingly, the same features of PDPN expression were also observed in our previous PDR study [8].

Notably, neoangiogenic mechanisms seemed to be activated in our hemi-RVO tissue specimen, since CD117 (c-kit) was expressed in the vessel-lining ECs. A previous experimental study had shown that a small subpopulation of CD117-positive ECs have proliferative capacity and the ability to differentiate toward functional ECs in adult blood vessel endothelium [12]. Indeed, according to that study, such a single c-kit-positive VESC could generate in vivo functional blood vessels that are capable of connecting to the host circulation [12]. Even though VESCs were known to display long-term self-renewal capacity, leading to the growth of a greater number of proliferative daughter cells in an experimental study, it was unclear whether this mechanism could be relevant also in advanced ischemic human hemi-RVO. Another possible explanation for our novel finding is that some end-differentiated mature retinal vascular wall ECs of our hemi-RVO eye were able to acquire stem cell-like characteristics in vivo by turning on the CD117 expression [12].

Our present work contradicts the current understanding that the intraocular posterior part of the human eye is devoid of lymphatics in ischemic hemi-RVO. Our study suggests that also in a severe sight-threatening fibrovascular RVO milieu, the antiangiogenic mechanisms can be overwhelmed and the physiological immune privilege broken, supporting our findings of lymphatic endothelial-like differentiation also in PDR [8]. In our study, the perfused, partially fibrotic neovascular tissue was situated at the optic nerve head. Since our IHC data verified the presence of vessel-like structures that stained with major LEC markers (LYVE-1 and Prox-1), we suggest that LECs might originate through sprouting from retinal venous BECs also in an advanced pathological RVO condition. Alternatively, tissue-resident and recruited circulating bone marrow-derived LEC progenitor cells such as macrophages or mesenchymal precursor cells could participate in the process of lymphatic-like differentiation in our pathological hemi-RVO case. In the future, to further investigate the development of RVO, double staining with LYVE-1+ or Prox-1+ CD68 + macrophages should be performed [15]. Unfortunately, in our study, we only rely on the expression of lymphatic and neoangiogenic markers/antigens, but have no functional data to prove our hypothesis. Unfortunately, no animal model is currently available to easily prove our hypothesis. However, recently, several in vivo and in vitro models of lymphangiogenesis have been developed. A further limitation of our study is that we have only examined one case with hemi-RVO. Therefore, we need to be careful of conclusions which have been based on only one sample. The lack of surgical patients suffering from this disease prevents us from receiving enough representative tissue material for the study.

Presently, it is acknowledged that active angiogenesis in RVO eyes leads to $\mathrm{VH}$, fibrosis formation, and ultimately to TRD, glaucoma, and visual loss. Thus far, the role of the lymphatic-like differentiation process in RVO has not been published. Currently, only limited evidence of efficient treatment options exists for RVO, with intravitreal injections of anti- 
Loukovaara et al.: A Case of Abnormal Lymphatic-Like Differentiation and Endothelial Progenitor Cell Activation in Neovascularization Associated with Hemi-RVO

VEGF agents emerging as a part of the first-line treatment, despite their potential, detrimental, long-term retinal side effects.

\section{Conclusion}

Our novel findings suggest that some lymphatic-like differentiation or BEC-to-LEC transdifferentiation occurs in the adult eye with hemi-RVO. This kind of plasticity between mature BEC and LEC phenotypes has been suggested by in vitro analyses more than a decade ago [3]. Our work suggests that the immune privilege in the posterior eye segment is devoid under a very advanced ischemic fibrovascular condition related to hemi-RVO. This finding is of great importance, considering the future treatment modalities for this devastating eye disease. Our current study suggests that in addition to angiogenic mechanisms, lymphatic and neoangiogenic mechanisms seem to be implicated in the hemi-RVO pathophysiology as well, suggesting that current treatment modalities such as anti-VEGF agents are and will not be efficient enough as therapeutic agents, but should potentially include the adjuvant anti-VEGFR-3 agents, since, generally, lymphangiogenesis can be inhibited by blocking the VEGF-C/VEGF-D/VEGFR-3 signaling pathway [5, 16]. Additionally, knowledge of the mechanisms regulating lymphatic-like differentiation and progenitor cell activity would be an important topic of further research in other devastating vitreoretinal ischemic eye disorders, and, therefore, functional in vitro or in vivo studies seem a great necessity in the nearby future as well. Our data also highlight the need to investigate vascular tube morphogenesis with ultrastructural analysis through transmission electron microscopy to unravel mechanisms underlying the pathological vascular hem- and lymphangiogenesis in RVO in more detail.

\section{Acknowledgements}

We thank the Biomedicum Molecular Imaging Unit for the imaging facilities. The study was supported by grants from the Academy of Finland (K.L., P.S.), the Sigrid Juselius Foundation (K.L., P.S.), the Finnish Eye Foundation (S.L.), the Finnish Cancer Organizations (K.L., P.S.), the Eye and Tissue Bank Foundation (S.L.), the Mary and Georg C. Ehrnrooth Foundation (S.L.), the Nissi Foundation (S.L.), the Friends of the Blind (S.L.), the Doctoral Program in Biomedicine (E.G.), and Helsinki University Central Hospital Clinical Research Grants (TKK4150 and TYH1325 to S.L.).

\section{Statement of Ethics}

The authors have no ethical conflicts to disclose.

\section{Disclosure Statement}

The authors report no conflicts of interest. 
Loukovaara et al.: A Case of Abnormal Lymphatic-Like Differentiation and Endothelial Progenitor Cell Activation in Neovascularization Associated with Hemi-RVO

\section{References}

1 Tuuminen R, Loukovaara S: Increased intravitreal angiopoietin-2 levels associated with retinal vein occlusion. Acta Ophthalmol 2014;92:e164-e165.

-2 Mäkinen T, Norrmen C, Petrova TV: Molecular mechanisms of lymphatic vascular development. Cell Mol Life Sci 2007;65:1915-1929.

-3 Petrova TV, Mäkinen T, Mäkela TP, Saarela J, Virtanen I, Ferrell RE, Finegold DN, Kerjaschki D, Ylä-Herttuala S, Alitalo K: Lymphatic endothelial reprogramming of vascular endothelial cells by Prox-1 homeobox transcription factor. EMBO J 2002;21:4593-4599.

4 Alitalo K: The lymphatic vasculature in disease. Nat Med 2011;17:1371-1380.

5 Nakao S, Hafezi-Moghadam A, Ishibashi T: Lymphatics and lymphangiogenesis in the eye. J Ophthalmol 2012;2012:783163.

6 Park D-Y, Lee J, Park I, Choi D, Lee S, Song S, Hwang Y, Hong KY, Nakaoka Y, Makinen T, Kim P, Alitalo K, Hong YK, Koh GY: Lymphatic regulator PROX1 determines Schlemm canal integrity and identity. J Clin Invest 2014;124:3960-3974.

7 Hos D, Schlereth SL, Bock F, Heindl LM, Cursiefen C: Antilymphangiogenic therapy to promote transplant survival and to reduce cancer metastasis: what can we learn from the eye? Semin Cell Dev Biol 2015;38:117-130.

8 Loukovaara S, Gucciardo E, Repo P, Vihinen H, Lohi J, Jokitalo E, Salven P, Lehti K: Indications of lymphatic endothelial differentiation and endothelial progenitor cell activation in the pathology of proliferative diabetic retinopathy. Acta Ophthalmol 2015, DOI: 10.1111/aos.12741.

-9 Banerji S, Ni J, Wang SX, Clasper S, Su J, Tammi R, Jones M, Jackson DG: LYVE-1, a new homologue of the CD44 glycoprotein, is a lymph-specific receptor for hyaluronan. J Cell Biol 1999;144:789-801.

$\$ 10$ Wigle JT, Oliver G: Prox1 function is required for the development of the murine lymphatic system. Cell 1999;98:769-778.

11 Baluk P, McDonald DM: Markers for microscopic imaging of lymphangiogenesis and angiogenesis. Ann NY Acad Sci 2008;1131:1-12.

12 Fang S, Wei J, Pentinmikko N, Leinonen H, Salven P: Generation of functional blood vessels from a single ckit+ adult vascular endothelial stem cell. PLoS Biol 2012;10:e1001407.

13 Kerrigan AM, Navarro-Nuñez L, Pyz E, Finney BA, Willment JA, Watson SP, Brown GD: Podoplaninexpressing inflammatory macrophages activate murine platelets via CLEC-2. J Thromb Haemost 2012;10:484-486.

14 Hong YK, Harvey N, Noh YH, Schacht V, Hirakawa S, Detmar M, Oliver G: Prox1 is a master control gene in the program specifying lymphatic endothelial cell fate. Dev Dyn 2002;225:351-357.

15 Schlereth SL, Neuser B, Caramoy A, Grajewski RS, Koch KR, Schrödl F, Cursiefen C, Heindl LM: Enrichment of lymphatic vessel endothelial hyaluronan receptor 1 (LYVE-1)-positive macrophages around blood vessels in the normal human sclera. Invest Ophthalmol Vis Sci 2014;55:865-872.

16 Tammela T, Zarkada G, Wallgard E, Murtomäki A, Suchting S, Wirzenius M, Waltari M, Hellström M, Schomber T, Peltonen R, Freitas C, Duarte A, Isoniemi H, Laakkonen P, Christofori G, Ylä-Herttuala S, Shibuya M, Pytowski B, Eichmann A, Betsholtz C, Alitalo K: Blocking VEGFR-3 suppresses angiogenic sprouting and vascular network formation. Nature 2008;454:656-659. 


\section{Case Reports in \\ Ophthalmology}

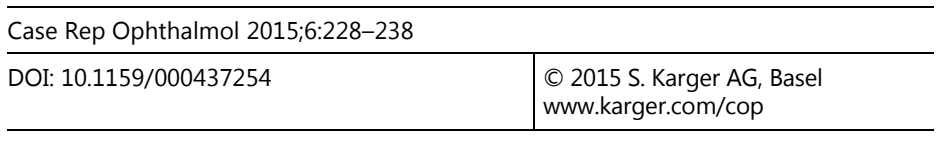

Loukovaara et al.: A Case of Abnormal Lymphatic-Like Differentiation and Endothelial Progenitor Cell Activation in Neovascularization Associated with Hemi-RVO

a H\&E

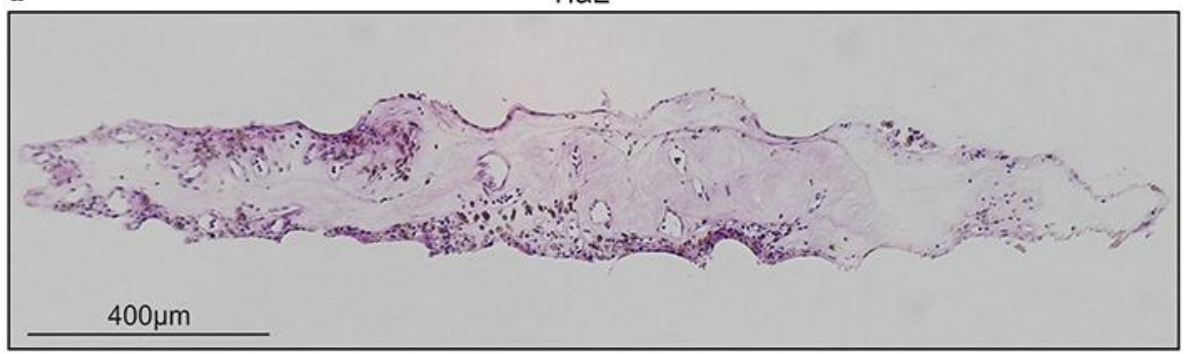

b CD31 a-SMA

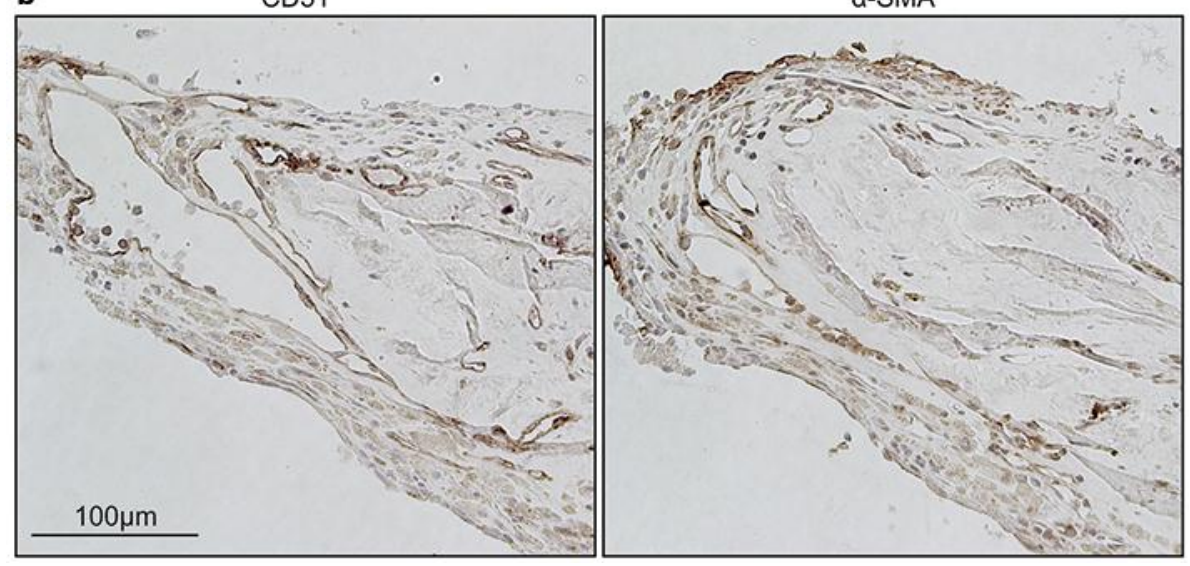

Fig. 1. Neovessel and fibrosis formation in the eye of a hemi-RVO patient. a H\&E staining of the whole tissue shows a fibrotic state and capillary-like structures. b Vascular ECs (CD31) and pericytes ( $\alpha$-SMA) lining the vessel structures were visualized from paraffin sections with IHC. 


\section{Case Reports in \\ Ophthalmology}

\begin{tabular}{l|l}
\hline \multicolumn{2}{l}{ Case Rep Ophthalmol 2015;6:228-238 } \\
\hline DOI: 10.1159/000437254 & $\begin{array}{l}\text { @ 2015 S. Karger AG, Basel } \\
\text { www.karger.com/cop }\end{array}$ \\
\hline
\end{tabular}

Loukovaara et al.: A Case of Abnormal Lymphatic-Like Differentiation and Endothelial Progenitor Cell Activation in Neovascularization Associated with Hemi-RVO
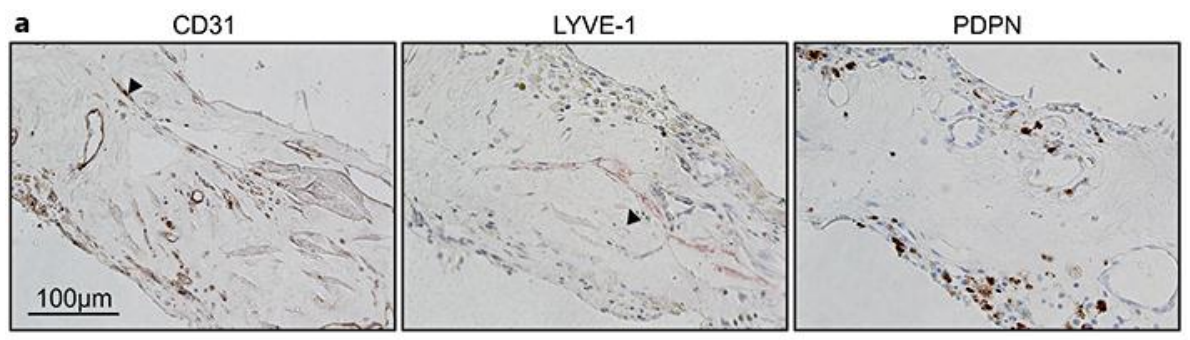

b
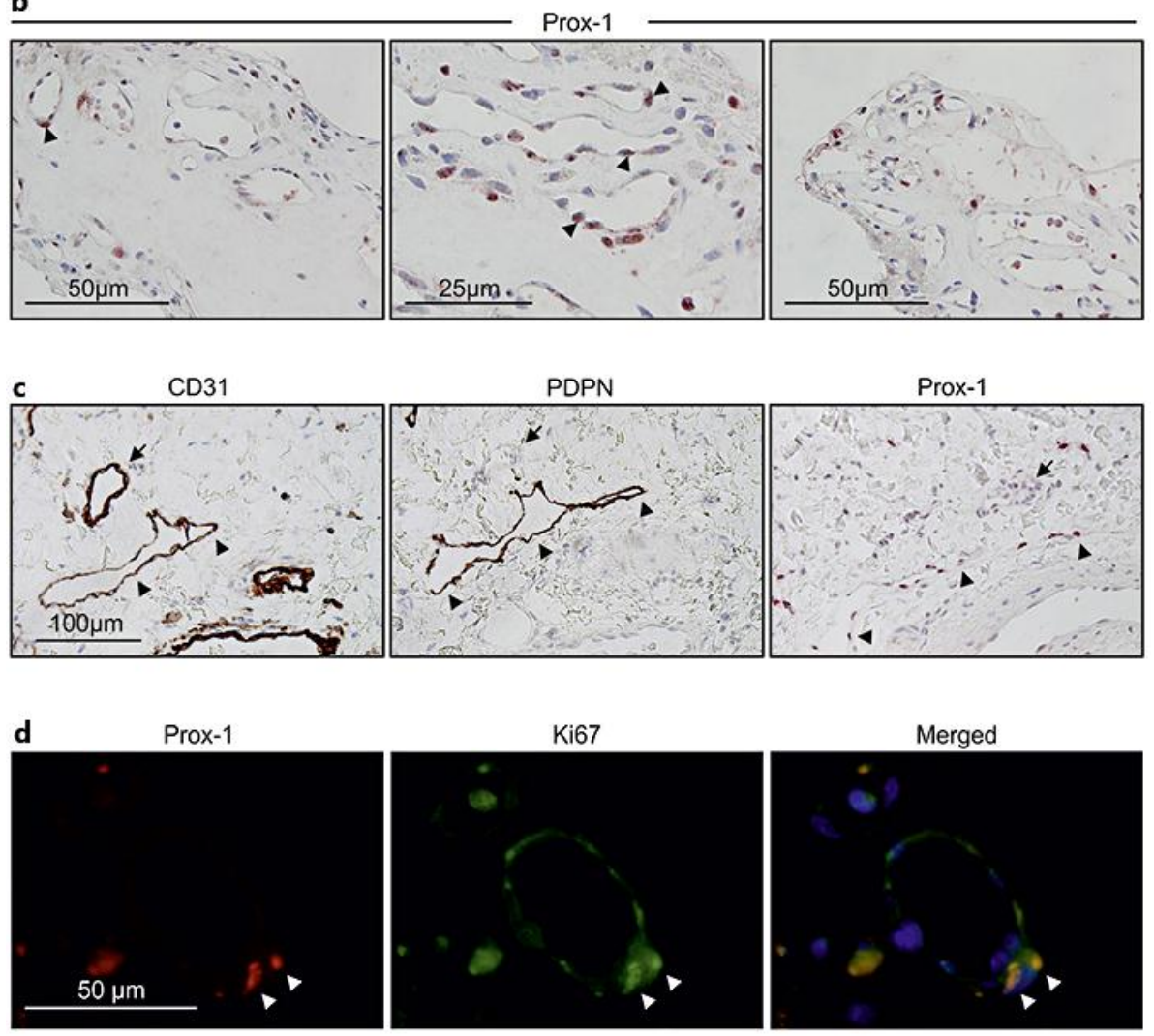

Fig. 2. LEC markers are expressed in neovascular hemi-RVO tissue specimen. a, b LYVE-1, PDPN, and nuclear transcription factor Prox-1 were used as markers for LECs. LYVE-1 immunoreactivity was observed (arrow head) (a, middle panel); immunoreactivity of PDPN was not found in any vascular structures, only in the extravascular structures (a, right panel), and corresponding pan-endothelial marker CD31 staining is also shown (arrow head) (a, left panel). Prox-1 positivity was observed in vessel-lining cells and also in circulating bone marrow-derived cells (arrow heads) (b). c Immunostaining of CD31, PDPN, and Prox-1 of the skin sample was used as control. Arrows indicate a blood vessel in the corresponding sections, and arrow heads indicate a lymphatic vessel showing positivity for PDPN and Prox-1. d Coimmunostaining of Prox-1 and Ki67 shows active proliferation of Prox-1-positive lumen-lining cells. 
Loukovaara et al.: A Case of Abnormal Lymphatic-Like Differentiation and Endothelial Progenitor Cell Activation in Neovascularization Associated with Hemi-RVO

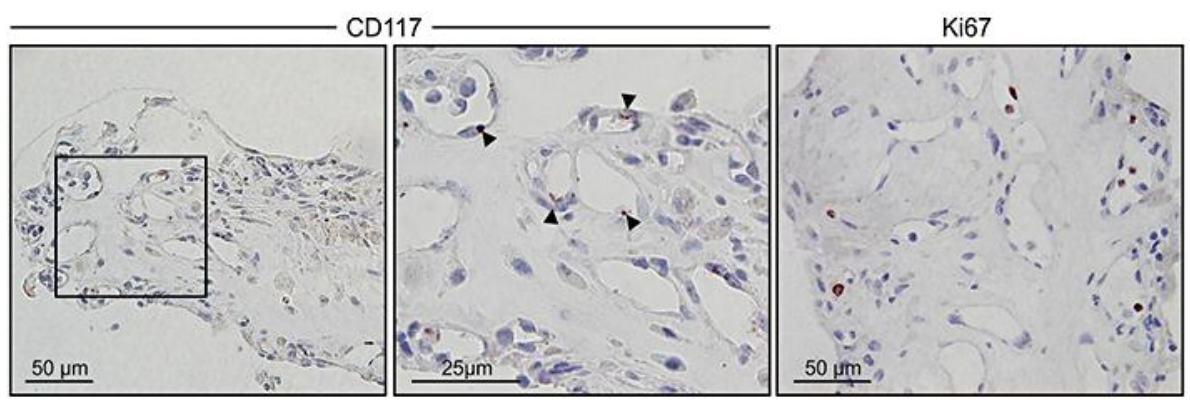

Fig. 3. Neovessel formation in hemi-RVO tissue involves vascular EC precursors. CD117 (c-kit) was used as a marker for VESCs (left panel, red). Larger magnification shows cytoplasmic staining (arrow heads) in lumen-lining vessel structures (middle panel). Immunoreactivity for the active proliferation marker Ki67 is also shown (right panel, red). 